\title{
Achromatic telescopic squeezing scheme and by-products: From concept to validation
}

\author{
S. Fartoukh $\odot,{ }^{*}$ M. Solfaroli, J. Coello De Portugalø, A. Mereghetti, \\ A. Poyet $\odot$, and J. Wenninger $\odot$ \\ CERN, Beams Department, CH 1211 Geneva 23, Switzerland
}

(Received 17 December 2020; accepted 21 January 2021; published 12 February 2021)

\begin{abstract}
The achromatic telescopic squeezing (ATS) scheme has brought an essential conceptual foundation to the HL-LHC project, making possible a strong and clean (achromatic) reduction of $\beta^{*}$, an important parameter with respect to which several HL-LHC sub-systems were dimensioned (e.g., the $150 \mathrm{~mm}$ aperture of the new inner triplet quadrupoles) or justified (crab-cavities for mitigating the geometric luminosity loss factor at low $\beta^{*}$ and subsequent large crossing-angle). The basic mechanics of the scheme is shortly reminded, highlighting as well some of its by-products (Landau damping, long-range beam-beam mitigation with octupoles), and a recent improvement which made possible an early, but still partial, implementation of ATS optics in the LHC for the 2017 and 2018 LHC physics runs. The main focus of the paper will be on the experimental validation of the scheme, via the development of dedicated machine configurations and high-intensity beam tests which took place in Run 2 . The paper will conclude on the configuration presently in mind to operate the LHC during its third exploitation period (Run 3), while trying to ensure a smooth enough transition toward the HL-LHC, in terms of optics, beam parameters, and dedicated beam manipulations $\left(\beta^{*}\right.$-leveling).
\end{abstract}

DOI: $10.1103 /$ PhysRevAccelBeams.24.021002

\section{INTRODUCTION AND MOTIVATIONS}

Reducing the beam sizes at the interaction point, namely acting on $\beta^{*}$ at constant beam emittances, is a key ingredient to boost the performance of any collider. Besides the need of larger-aperture magnets in order to safely operate the machine at lower $\beta^{*}$, the first challenge is to design the optics, that is to match it to the (nonupgraded) part of the ring, while preserving its flexibility within the matching quadrupole strength limits, and its chromatic properties in terms of off-momentum $\beta$-beating, linear and nonlinear chromaticities, and spurious dispersion induced by the crossing bumps (see [1] for more detail). The achromatic telescopic squeezing (ATS) scheme [2-4] brought a definite and cost-efficient solution to this problem.

\section{A. The basic mechanics}

This scheme is essentially based on a two-stage reduction of $\beta^{*}$ (squeeze). In a first step, a so-called presqueeze is achieved by using exclusively, as usual, the matching quadrupoles of the high-luminosity insertions IR1 and IR5,

\footnotetext{
*stephane.fartoukh@cern.ch
}

Published by the American Physical Society under the terms of the Creative Commons Attribution 4.0 International license. Further distribution of this work must maintain attribution to the author(s) and the published article's title, journal citation, and DOI. but imposing new constraints on the left and right betatron phase of these two insertions, till hitting some hard limits (matching quadrupole and/or sextupole strength limits). These limits are reached at $\beta^{*}=36 \mathrm{~cm}$ for the LHC [5], and $48 \mathrm{~cm}$ for the HL-LHC [6] due to the new longer triplets operating at lower gradient (but with larger aperture). In a second step, a so-called tele-squeeze further reduces $\beta^{*}$ by acting only on the matching quadrupole located in the insertions on either side of IR1 and IR5 (i.e., IR8/2 for the tele-squeeze of IR1, and IR4/ 6 for IR5). As a result, sizable $\beta$-beating bumps are induced in the four sectors on either side of IP1 and IP5, the so-called ATS sectors. These waves of $\beta$-beating are then also necessary in order to preserve the chromatic correction of the triplet, at constant strength by the lattice sextupoles located in the four ATS sectors (only one family used per beam, per sector and per plane, out of the two available in the LHC arcs). This essentially forms the basic idea behind the ATS scheme, a sort of generalized optics-squeezing scheme, involving up to $50 \%$ of the ring, and being found so far to be the only way to build collision optics with decimetric $\beta^{*}$ values, as requested by the HL-LHC (see Fig. 1). The scheme not only works to deliver round, but also flat collision optics (the primary target of the ATS scheme [2]), with an even more aggressive $\beta^{*}$ in one the two transverse planes.

In a given plane, and a given high-luminosity insertion (IR1 or IR5), the telescopic index (or tele-index) $r^{\text {Tele }}$ is 


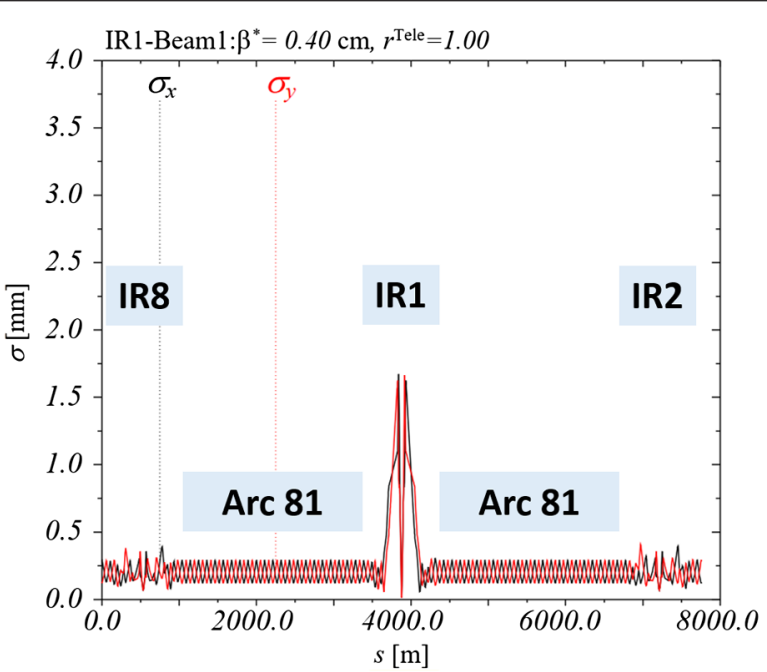

Pre-squeeze to $\beta^{*}=40 \mathrm{~cm}$

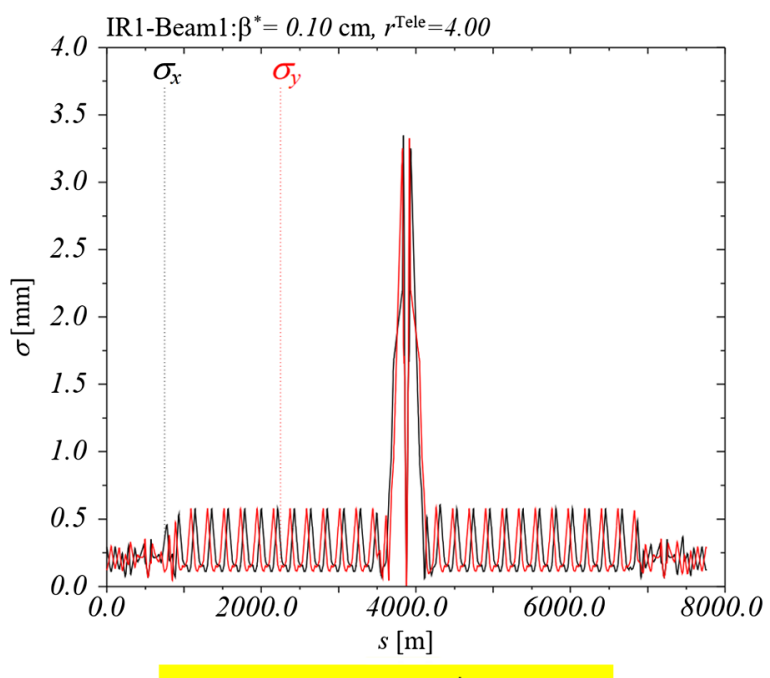

Tele-squeeze to $\beta^{*}=10 \mathrm{~cm}$

FIG. 1. Beam sizes [mm] at $7 \mathrm{TeV}$ along a quarter of the LHC ring for a typical ATS presqueezed (left) and tele-squeezed (right) optics. The interaction point (IP) of the ATLAS experiment is exactly at the center. In the end of the tele-squeeze the spot size is not larger than $6 \mu \mathrm{m}$ at the IP, thanks to the ATS scheme transforming $7 \mathrm{~km}$ of machine into a giant final focus system with natural chromatic correction sections embedded in Arc81 and Arc12.

defined as the ratio between the presqueezed $\beta^{*}$, and the actual value of $\beta^{*}$ at the IP, namely $\beta_{\text {Pre }}^{*}$ and $\beta_{\text {Tele }}^{*}$, respectively:

$$
r^{\text {Tele }} \stackrel{\text { def }}{=} \frac{\beta_{\text {Pre }}^{*}}{\beta_{\text {Tele }}^{*}}
$$

This quantity also gives the relative increase of the peak $\beta$-functions which arise in the four ATS sectors. The principle can also be used to de-squeeze $\beta^{*}\left(r^{\text {Tele }}<1\right)$, in which case the phase of the above-mentioned $\beta$-beating wave is just shifted by $\pi / 2$, i.e., one FODO cell. The usefulness of antitelescopic optics will be introduced later, in the context of Run 3 (see section III). The ATS scheme has of course its own limits in terms of tele-index reach (matching quadrupole strength limits in IR8/2/4/6), typically $1 / 3 \lesssim r^{\text {Tele }} \lesssim 4$ for telescopic round optics (i.e., with the same tele-index in both transverse planes, starting from a round presqueezed optics).

The presqueeze (IR1/5 settings) and tele-squeeze (IR2/8/4/6 settings) sequences can be combined or interleaved arbitrarily, as soon as specific left and right phase advance constraints can be matched in IR1 and IR5, in practice as soon as the presqueezed $\beta^{*}$ reaches a value in the range of $2-3 \mathrm{~m}$ (starting from $11 \mathrm{~m}$ for LHC at injection, and from $6 \mathrm{~m}$ for HL-LHC). This modularity opens in particular the possibility to deploy the telescope (or anti-telescope), fully or partially, already in the ramp, thus extending the concept of combined ramp and squeeze (CRS, as implemented in LEP and LHC $[7,8]$ ), to a so-called combined ramp and double squeeze (CRDS), i.e., with $r^{\text {Tele }} \neq 1$ at the end of the ramp (EoR). This gymnastic may not be necessarily driven by a smaller EoR $\beta^{*}$ (e.g., to maximize the operation efficiency), but by Landau damping of transverse coherent instabilities without risking to run out of strength in the arc octupoles (MO), as discussed hereafter.

\section{B. The ATS by-products}

The main by-products of ATS optics result from the $\beta$-beating waves induced in the four ATS sectors. In this configuration, not only the lattice sextupoles are boosted for the chromatic correction of the triplet, but also the lattice octupoles.

At large enough telescopic index (typically $r^{\text {Tele }} \sim 3-4$ ), $50 \%$ of the lattice octupoles located in the four ATS sectors become so efficient that they can mitigate the octupolarlike component of the long-range beam-beam (BBLR) interactions (see sketch in Fig. 2). The motivation is obviously to push the BBLR limit in terms of minimum possible crossing angle, in particular to operate the machine with alternated flat optics in IR1 and IR5, while sticking to a normalised crossing angle in the range of $\sim 9-12 \sigma$ (as for round optics, depending on the bunch charge from 1 to $2 \times 10^{11} \mathrm{p} / \mathrm{b}$ ). For flat optics, the crossing angle, deployed in the plane of largest $\beta^{*}$, becomes in this case substantially smaller in $\mu \mathrm{rad}$ compared to round optics, which strongly mitigates the geometric loss factor [9].

At a milder telescopic index $\left(r^{\text {Tele }} \sim 2\right.$ ), (anti-)telescopic optics represents as well an elegant solution for Landau damping brighter and/or more energetic beams, without running the MOs out of strength, especially at flat-top 


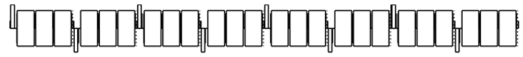

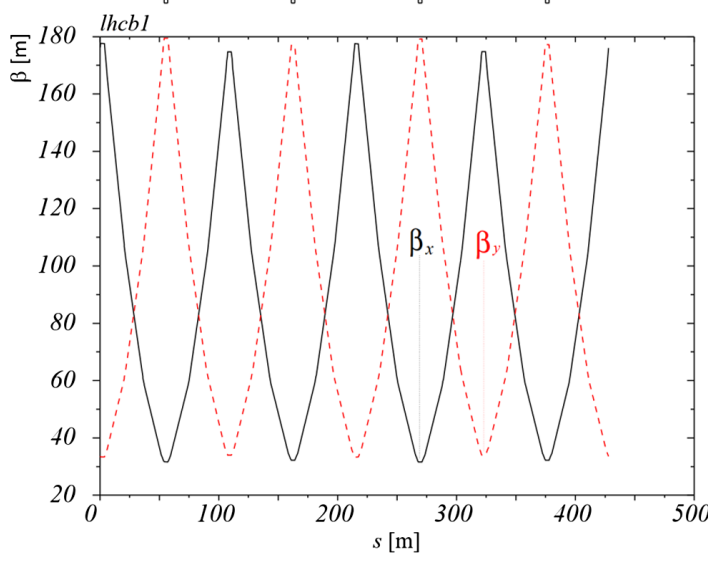

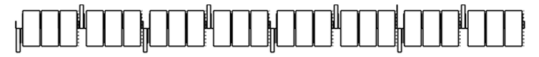

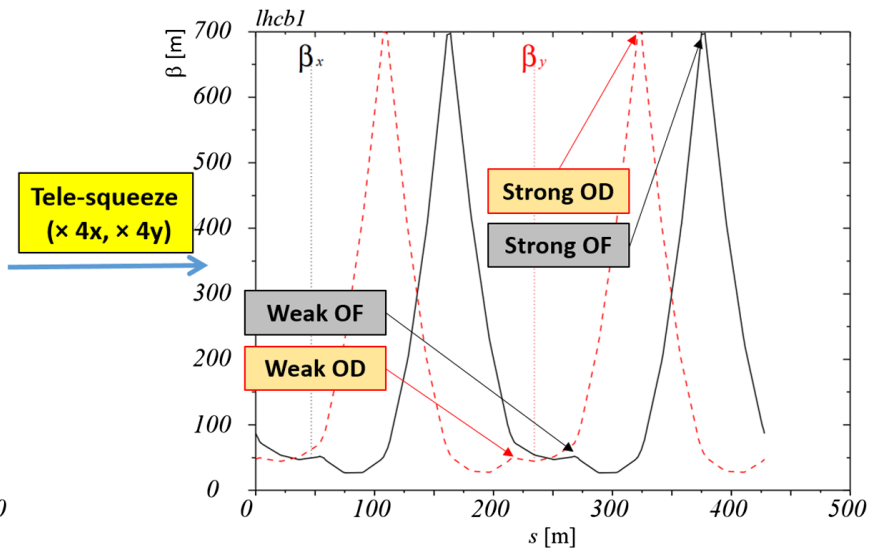

FIG. 2. Deformation of the LHC arc optics by the action of the tele-squeeze, which splits the lattice octupoles (one circuit per beam, per arc and per plane in the LHC) into two optically different families, the "strong" and the "weak" MOs. In a given plane, the strong MOs are located at the peak of the corresponding $\beta$-beating wave, that is at $k \pi$ with respect to the triplet and, de facto, correctly phased with respect to the long-range beam-beam interactions. For anti-telescopic optics, weak and strong MOs are simply exchanged.

energy where the demand is the highest (see example in Fig. 3). Deploying the telescope in the ramp seems therefore to be a natural direction where to go in Run 3, when only a fraction of the collimators will have been replaced with new ones of lower impedance.

Several experimental evidences of these by-products were obtained through high-intensity beam tests in Run 2, which forms the main subject of the next section.

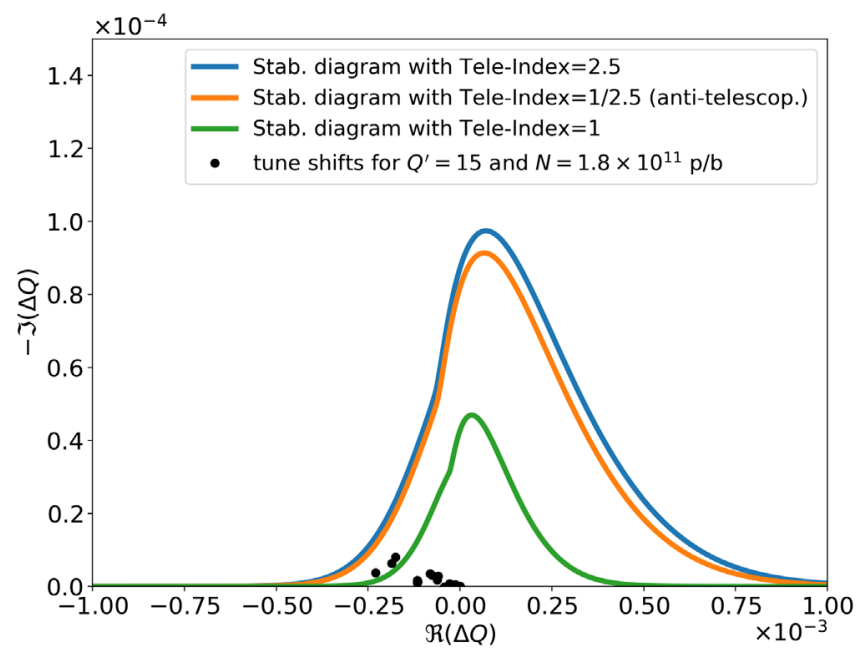

FIG. 3. Typical improvement of the beam stability diagram (SD) thanks to (anti-)telescopic optics at constant MO current (350 A with positive polarity), assuming a linear chromaticity of $Q^{\prime}=15$ units (as in Run 2). The coherent modes are superimposed, assuming a bunch population of $1.8 \times 10^{11} \mathrm{p} / \mathrm{b}$ within a normalized emittance of $\gamma \epsilon=1.8 \mu \mathrm{m}$, as calculated from the strict LHC Run 3 impedance model and multiplying the resulting coherent tune shifts by an extra empirical factor of 2 (as observed in Run 2 [10]). Courtesy of N. Mounet.

\section{IMPLEMENTATION IN THE LHC AND SPECIAL TESTS IN RUN 2}

\section{A. Gradual implementation of ATS optics in the LHC}

The telescopic mechanics of the ATS scheme, together with its achromatic properties, were demonstrated in Run 1 using small intensity beams [11]. These tests culminated in 2012 with the demonstration of a $10 \mathrm{~cm}$ telescopic optics [12], of course in non-nominal conditions due to the aperture restriction of the existing triplet. At that time, and till very recently, all LHC and HL-LHC ATS optics versions however led to very unfavorable horizontal betatron phase advances, nearly equal to 90 degrees, between the extraction kickers (MKD) in the dump insertion (IR6) and some tertiary collimators (TCT) in IR1 and IR5, in particular the most exposed one in case of asynchronous dump (TCT.R5B2). When discussing the possibility to directly use ATS optics in order to restart the LHC for 6.5 TeV operation in 2015, this feature was shown to be a clear weakness of the scheme, which rapidly discarded this option. A new generation of ATS optics was then worked out in order to bring a definite cure to the above mentioned limitation, offering phase advances very close to optimal (within a specified tolerance of $30^{\circ}$ [13]) between the MKDs and TCTs, for both beams and both IR1 and IR5 (see [14] for more detail). This enabled first ATS tests to take place in 2016 with unsafe beams (a few nominal bunches), still at a rather modest telescopic index $\left(\beta^{*}=33 \mathrm{~cm}\right.$ corresponding to $r^{\text {Tele }} \approx 1.2$ for a presqueezed $\beta^{*}$ adjusted to $40 \mathrm{~cm}$ ), in order to start validating the optics on other aspects, in particular collimation (see Fig. 4). Stepping back to low intensity beams, the correct-ability of this new ATS optics version was then (re-)demonstrated at a more sizable telescopic index of $2\left(\beta^{*}=21 \mathrm{~cm}\right)$, together 


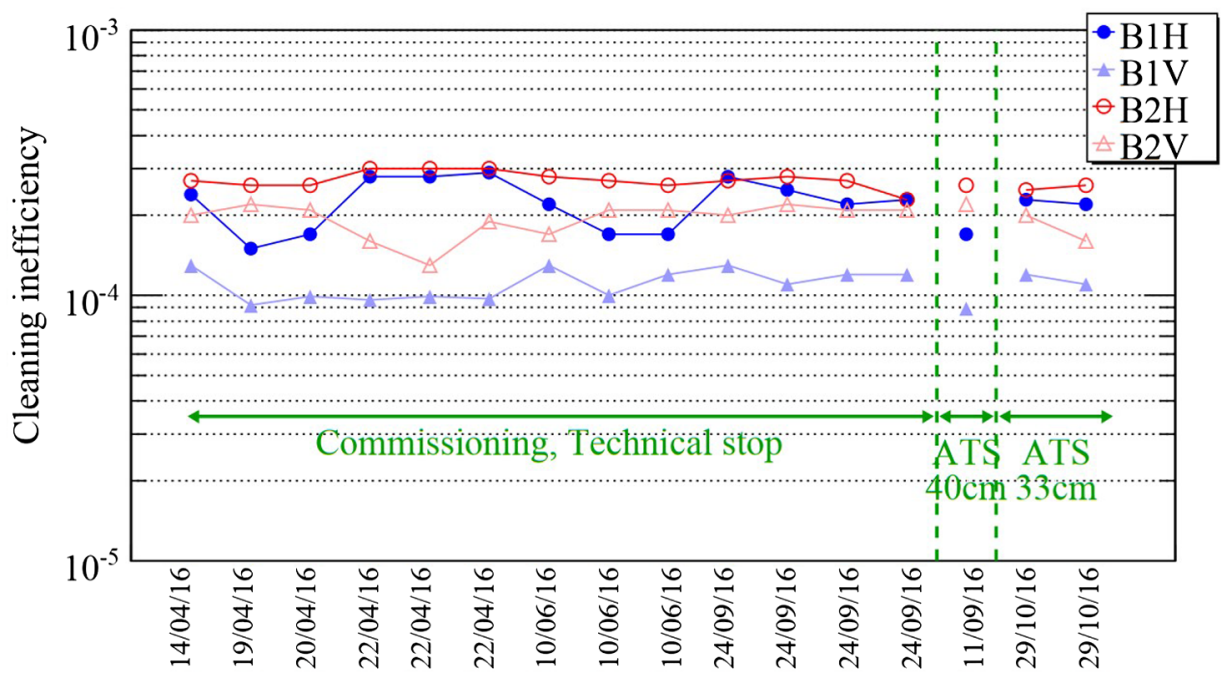

FIG. 4. Collimation inefficiency measured over the 2016 LHC run (non-ATS optics), and during the 2016 ATS machine development sessions. Courtesy of D. Mirarchi [14].

with its clean chromatic properties in terms of Montague functions (see Fig. 5).

Completing the beam tests with dedicated simulation studies, in particular beam-beam studies [15], the decision was taken to use this new ATS optics version for the 2017 LHC luminosity run. Starting from a $40 \mathrm{~cm}$ presqueezed optics, the telescopic squeezing techniques were then used to push $\beta^{*}$ down to $30 \mathrm{~cm}$ in 2017 (i.e., corresponding to $r^{\text {Tele }}=1.33$ ). In 2018 , it was decided to further reduce $\beta^{*}$ from $30 \mathrm{~cm}$ down to $25 \mathrm{~cm}\left(r^{\text {Tele }}=1.6\right)$ with the beams colliding (so-called " $\beta^{*}$-levelling"), after a first period of crossing-angle antileveling at $\beta^{*}=30 \mathrm{~cm}$ in order to liberate enough aperture in the inner triplet (see e.g., [16] for a more thorough description of the LHC machine configurations in Run 2). Detailed comparisons between the 2016 and 2017 physics runs, in particular in terms of optics correct-ability, collimation inefficiency, and beam lifetime, did not reveal any striking differences between the previous nominal LHC optics and the just implemented ATS optics, but still at a rather modest telescopic index (see [17] for an overview).

\section{B. High-intensity beam tests with HL-LHC-like round optics}

In order to validate the full ATS scheme and its by-products with bunch trains, two special machine configurations were developed and tested in machine development (MD) periods, with HL-LHC like telescopic indexes $\left(r^{\text {Tele }} \approx 3\right)$, while keeping a collision $\beta^{*}$ compatible with the aperture of the existing triplet $\left(\beta_{\text {Tele }}^{*} \gtrsim 30 \mathrm{~cm}\right.$ ), namely by adjusting (increasing) the presqueezed $\beta^{*}$ accordingly.

The first ATS MD program (2017) was based on the nominal LHC ramp (ending up at $\beta^{*}=1 \mathrm{~m}$ in 2017-2018), immediately followed by a tele-squeeze to reach a $\beta^{*}$ of
$35-25 \mathrm{~cm}\left(r^{\text {Tele }} \approx 3-4\right)$. The high-intensity beam tests took place at $\beta_{\text {Tele }}^{*}=35 \mathrm{~cm}$ where, in particular, loss map measurements did not show any anomalies, thus demonstrating for the first time the viability of highly telescopic optics from the perspective of the collimation system. A rather good optics correctability was also demonstrated, but with indications that a telescopic index of 3 is at the level where some new types of imperfections (internal to the arcs) start to show up, requesting new $\beta$-beating correction techniques to go beyond (in particular for flat optics, see next section). When a few trains were put into collision, no beam lifetime nor emittance growth issues were observed, the luminosity was found as expected (see [18] for more detail). The program ended up with the demonstration of the possible mitigation of the BBLR interactions via the lattice octupoles (see Fig. 6), as announced in Sec. IB for ATS optics with sufficiently high telescopic indexes.

The second ATS MD program (2018) was based on a new ramp variant (CRDS), with the tele-squeeze fully embedded in the ramp (see Sec. I A). In the first part of the ramp, the presqueezed $\beta^{*}$ was already pushed down to $2 \mathrm{~m}$ when reaching $2.7 \mathrm{TeV}$, based on which a dedicated tele-squeeze sequence followed, to end up the ramp with a $\beta^{*}$ of $65 \mathrm{~cm}$, that is with a telescopic index of $r^{\text {Tele }}=2 / 0.65 \approx 3.1$. In addition to the operational demonstration of this new type of ramp, a series of typical activities was successfully conducted (optics correction illustrated in Fig. 7, loss map measurements at flat-top energy and taken on the fly during the ramp), in order to validate this new machine configuration for high-intensity beams. Also dedicated studies took place, related to collective effects. After injecting a few nominal bunches $\left(1.1-1.2 \times 10^{11} \mathrm{p} / \mathrm{b}\right)$, the new ramp was played several times, using $\mathrm{MO}$ current ramping functions typically twice 

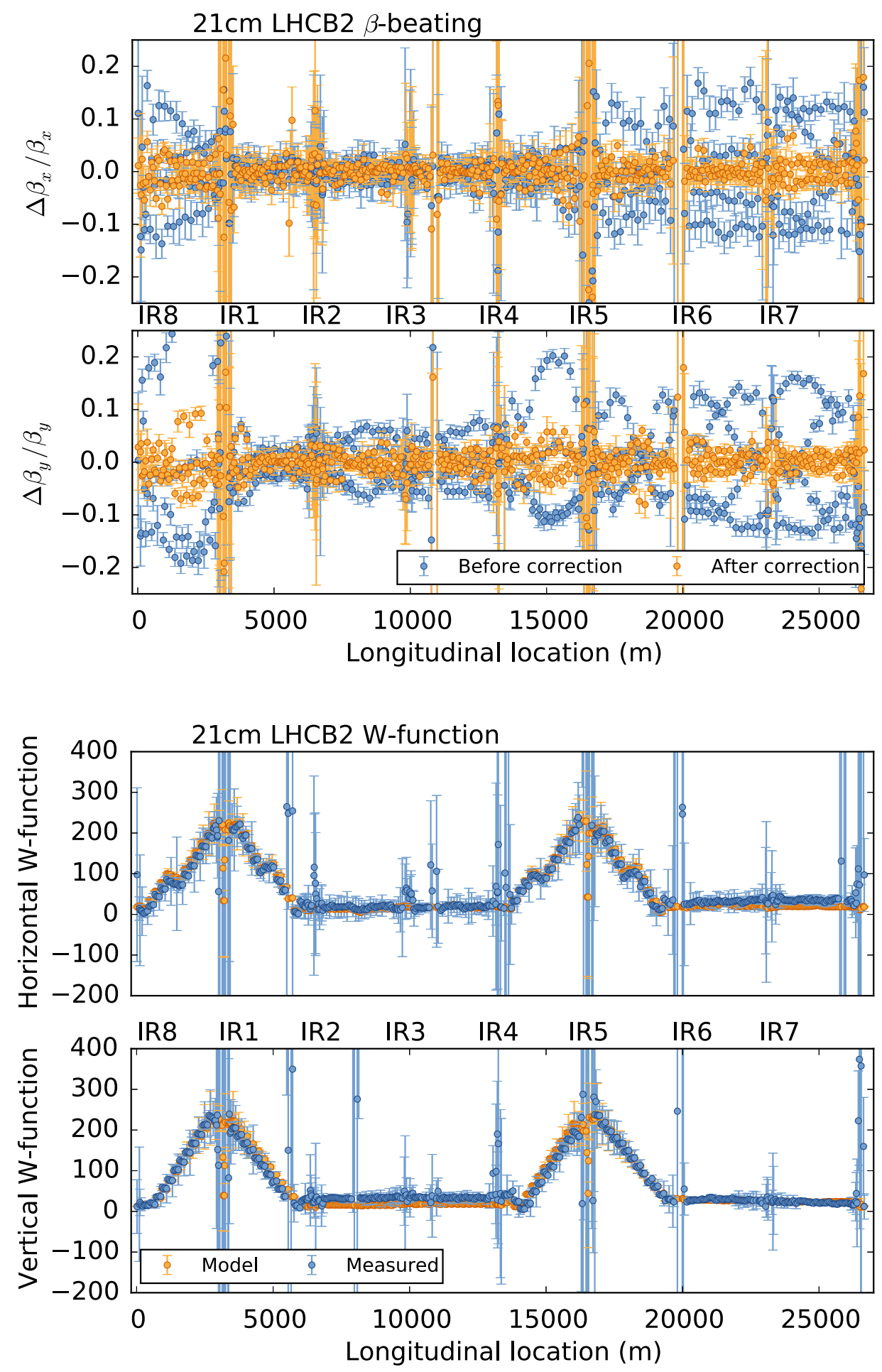

FIG. 5. 2016 ATS MD: $\beta$-beating $(\mathrm{H} / \mathrm{V})$ for Beam 2 at $\beta^{*}=21 \mathrm{~cm}\left(r^{\text {Tele }} \approx 2\right)$ before and after correction (top), and corresponding chromatic functions (bottom).

less than nominal (200 A EoR), with either polarity, and demonstrating at flat-top energy quite small MO thresholds before instability (see Fig. 8), as expected from the large telescopic index (see Section I B). The intensity ramp up was fast and successful. It culminated with about
750 nominal bunches packed into 48 bunch trains $\left(\sim 1.2 \times 10^{11} \mathrm{p} / \mathrm{b}\right)$ in a first test and, later on, injecting high-intensity $8 \mathrm{~b} 4 \mathrm{e}$ bunch trains [19] in this new machine configuration ( 800 bunches with $\sim 1.6 \times 10^{11} \mathrm{p} / \mathrm{b}$ ). Figure 9 compares the CRDS to the nominal ramp in terms of beam 


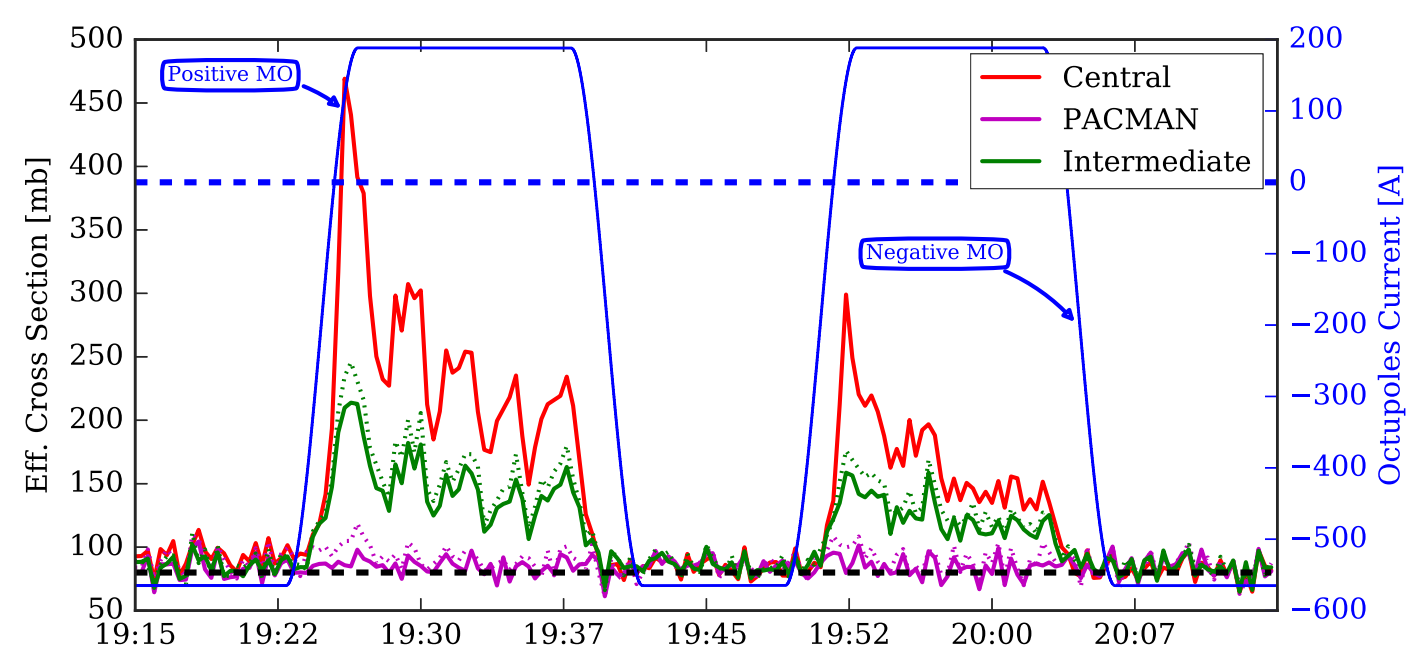

FIG. 6. 2017 ATS MD: lifetime evolution for various individual bunches of Beam 1 when scanning the MO current in collision at $\beta_{\text {Tele }}^{*}=35 \mathrm{~cm}\left(r^{\text {Tele }} \approx 3\right.$ ), with an aggressive crossing angle of $240 \mu \mathrm{rad}$ (full). During the scan, the average bunch population of Beam 2 was measured in between $1.0 \times 10^{11}$ and $0.95 \times 10^{11} \mathrm{p} / \mathrm{b}$. The lifetime is expressed in terms of so-called effective cross-section, i.e., loss rate normalised by luminosity, $\sigma_{\text {eff }_{i}} \stackrel{\text { def }}{=}\left|d N_{i} / d t\right| / \mathcal{L}_{i}$. Pacman and intermediate bunches were selected to experience only half or three quarters of the BBLR encounters (two bunches for each case, in dashed and solid lines, located at the head and at the tail of the first bunch train), compared to the central bunch (solid red line) undergoing the maximum possible number of BBLR collisions. Reverting the MO polarity, the burn-off limit of $80 \mathrm{mb}$ (total inelastic proton cross-section at $6.5 \mathrm{TeV}$ ) was reached for all classes of bunches.

lifetime, as recorded during the $8 \mathrm{~b} 4 \mathrm{e}$ tests for two consecutive fills where the machine configuration was changed in between. The lifetime of Beam 2 was found to be sensibly worse at the end of the CRDS compared to the nominal ramp, but still in the range of $50-100 \mathrm{~h}$, i.e., similar in absolute to Beam 1 for both ramps. 8b4e bunch trains were also successfully put in collision immediately at the end of the CRDS at $\beta^{*}=65 \mathrm{~cm}$, and the burn-off limit

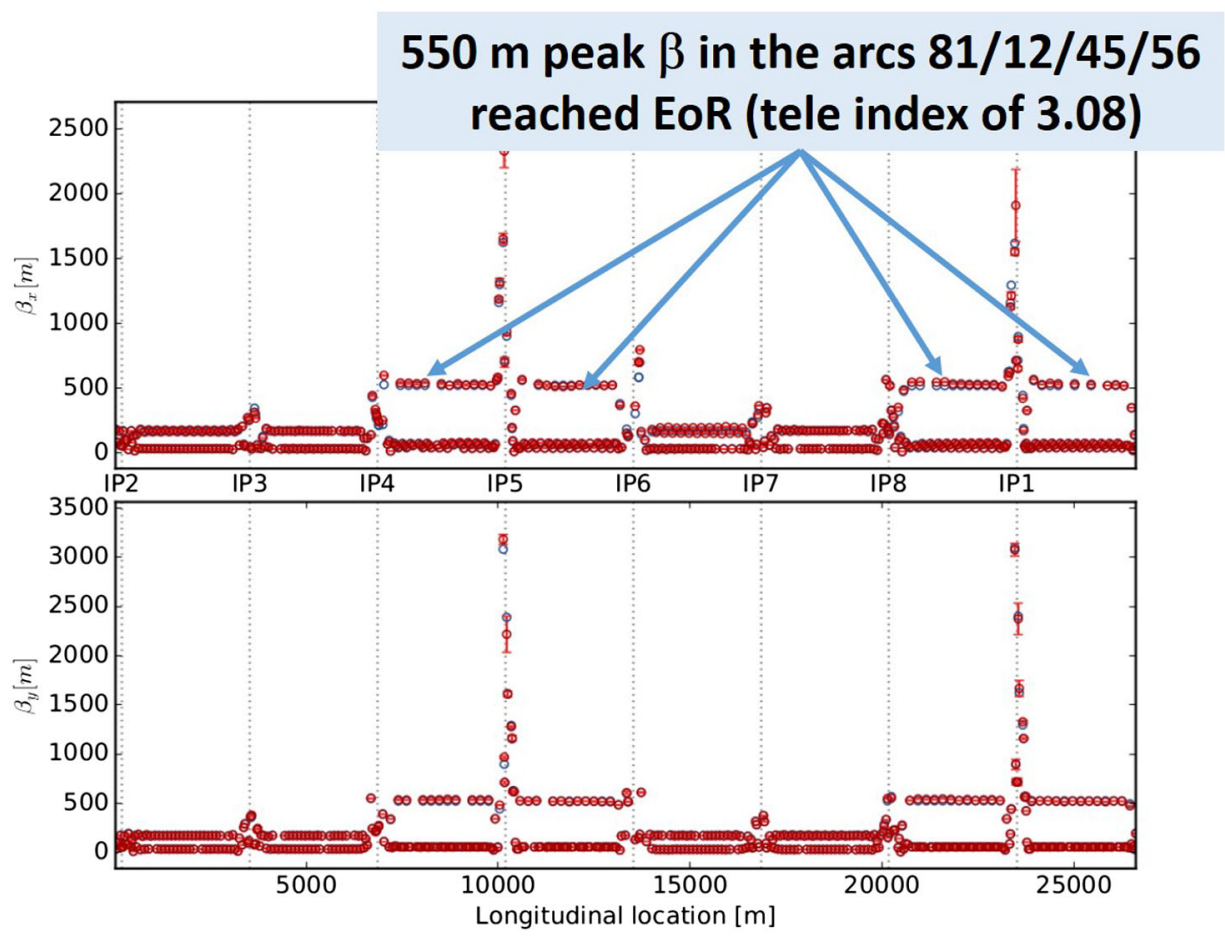

FIG. 7. 2018 ATS MD: optics measurement after correction at the end of the CRDS: $r^{\text {Tele }}=3.08\left(\beta_{\text {Tele }}^{*}=65 \mathrm{~cm}\right)$, leading to an effective tune spread almost tripled at constant MO current. 


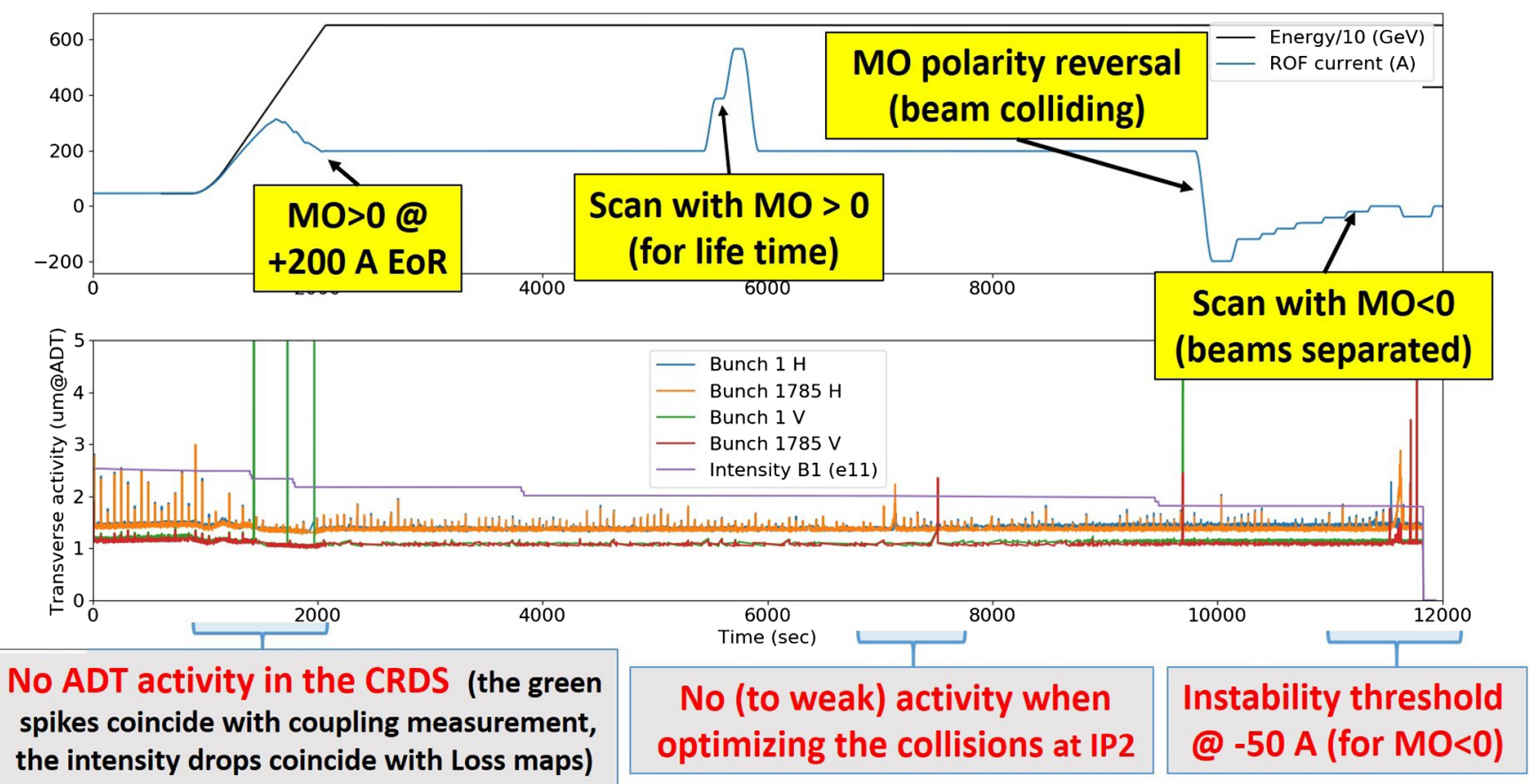

FIG. 8. 2018 ATS MD: MO ramp function (in the case of positive polarity), Beam 1 activity observation in the ramp, i.e., turn by turn oscillation amplitude at a dedicated highly sensitive pick-up nearby the transverse damper (ADT), and measurement of the MO threshold at $6.5 \mathrm{TeV}$ with negative polarity $\left(\sim 1.1 \times 10^{11} \mathrm{p} / \mathrm{b}\right)$. For this measurement, the machine was filled with a series of pilot bunches $\left(\sim 6-7 \times 10^{9} \mathrm{p} / \mathrm{b}\right)$ for loss maps measurements, and two nominal bunches $\left(\sim 1.0 \times 10^{11} \mathrm{p} / \mathrm{b}\right)$, namely bunch 1 and 1785 for Beam 1, for the measurement of the MO instability threshold at flat top energy.
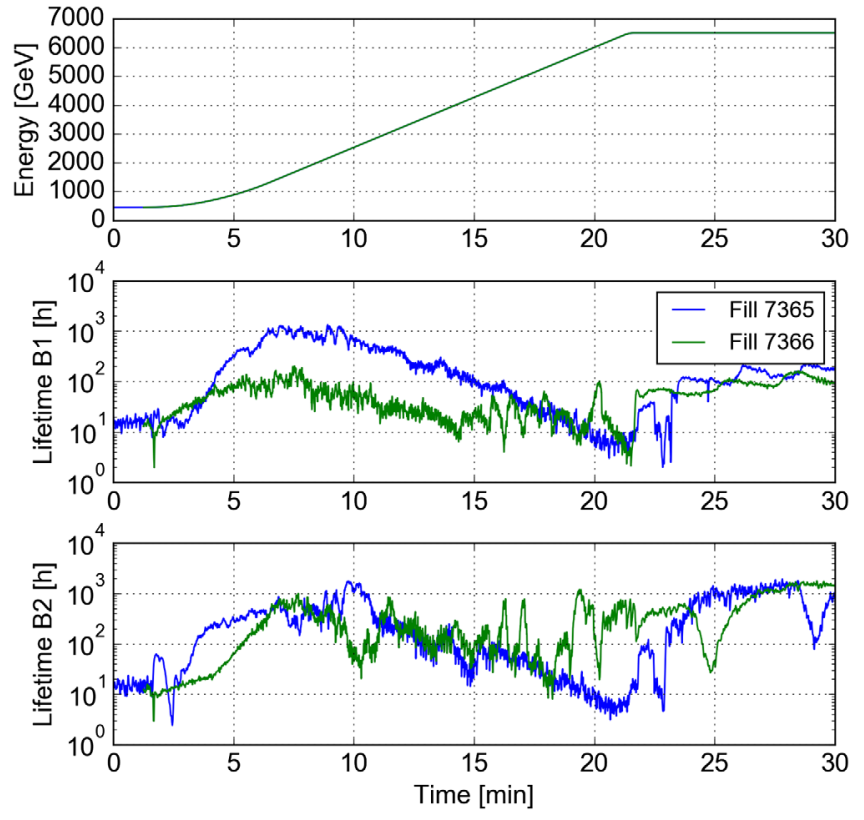

FIG. 9. 2018 ATS MD: beam lifetime with 8b4e trains $\left(\sim 1.6 \times 10^{11} \mathrm{p} / \mathrm{b}\right.$ wit $\left.\gamma \epsilon \sim 3 \mu \mathrm{m}\right)$ for the CRDS (Fill 7365 with 800 bunches) and the nominal CRS (Fill 7366 with 1600 bunches). Courtesy of G. Iadarola [18]. was demonstrated after a tune scan $\left(\Delta Q_{x / y}=0.007 / 0.005\right.$, see Fig. 10). More details can be found in [20].

\section{Flat telescopic optics and first high-intensity beam test}

Flat optics have been the primary motivation for the development of the ATS scheme [2], targeting a very aggressive $\beta^{*}$ in the range of the r.m.s. bunch length $\left(\beta_{\|}^{*} \sim \sigma_{z} \sim 7.5 \mathrm{~cm}\right)$ in one of the two transverse planes (the parallel separation plane), while deploying the crossing angle in the plane of largest $\beta^{*}$ (for triplet aperture preservation), and with a typical $\beta^{*}$ aspect ratio of 4 , namely $r^{*} \stackrel{\text { def }}{=} \beta_{X}^{*} / \beta_{\|}^{*} \sim 4$. Flat optics supported by appropriate techniques to mitigate the BBLR interactions, indeed offers a very competitive alternative (or a complement) to the HL-LHC baseline [21,22], which otherwise only relies on crab-cavities to mitigate the geometric luminosity loss factor. Accordingly, a third and last special ATS hypercycle was built up in Run 2, for high-intensity beam tests using a flat optics with $\beta_{\|}^{*}=15 \mathrm{~cm}$ at IP1 and IP5 in the vertical and horizontal planes, respectively, and $\beta_{X}^{*}=60 \mathrm{~cm}$ in the other (crossing) plane (swapped with respect to the nominal LHC configuration in order to liberate enough triplet aperture in the plane of smallest $\beta^{*}$ [9]). This collision optics was based on the nominal $60 \mathrm{~cm}$ presqueezed optics, 

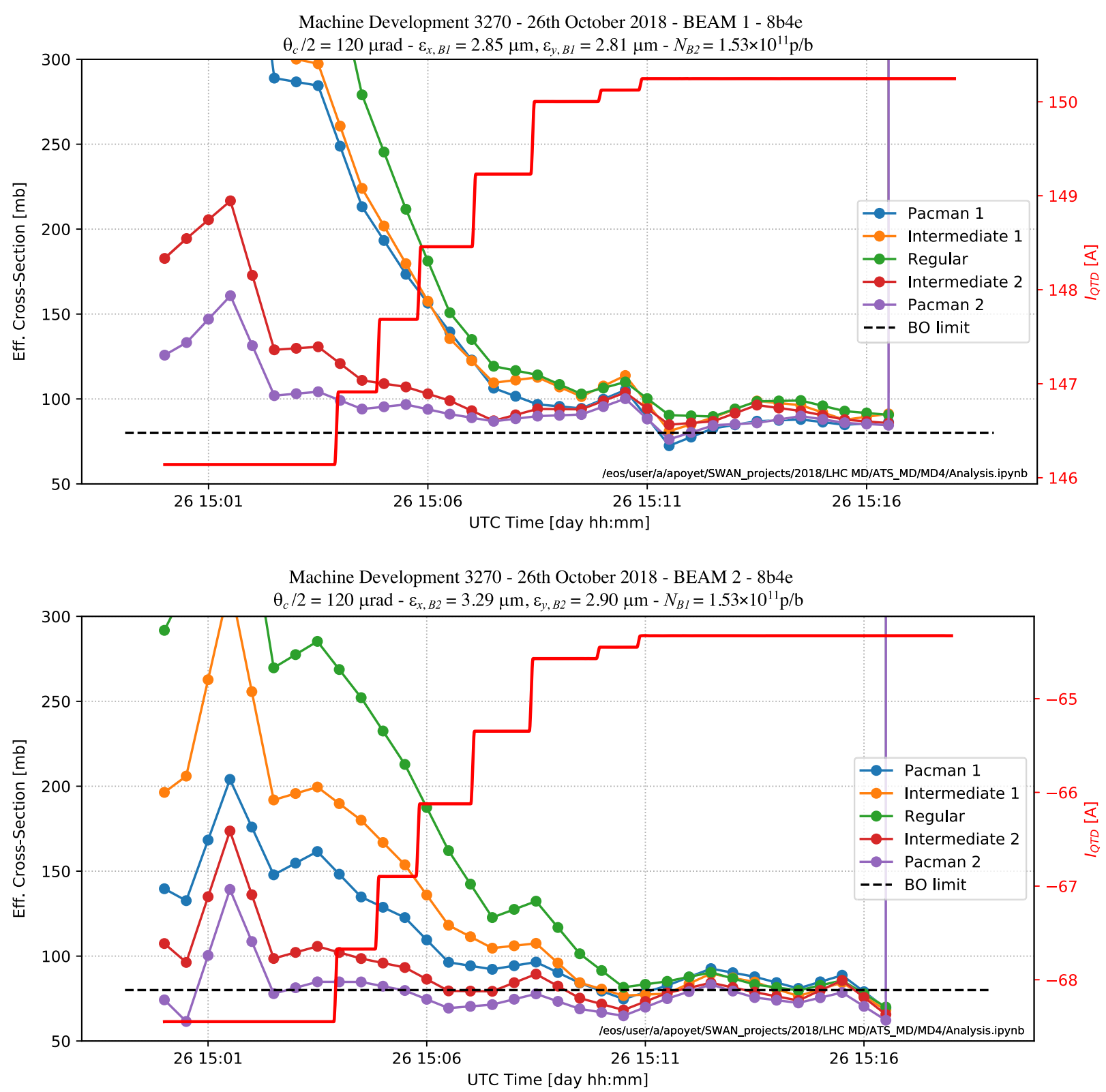

FIG. 10. 2018 ATS MD: 8b4e bunch trains in collision at $\beta_{\text {Tele }}^{*}=65 \mathrm{~cm}\left(\theta_{c} / 2=120 \mu \mathrm{rad}\right)$. The burn-off limit was reached for various classes of bunches of Beam 1 (top) and Beam 2 (bottom) after a tune scan along the diagonal, and with the octupoles pushed to their maximum current of $550 \mathrm{~A}$ with negative polarity.

therefore with a telescopic index adjusted to $r_{\|}^{\text {Tele }}=4$ in the plane of smallest $\beta^{*}$, and kept to $r_{X}^{\text {Tele }}=1$ (no telescope) in the other plane. The operational mechanics of this hypercycle was fully demonstrated, including the rotation of the crossing planes in ATLAS and CMS performed in the end of the ramp (see Fig. 11). The optics correction was found sensibly more difficult compared to the round optics case, not necessarily due to the optics flatness, but due to the very low $\beta^{*}$ of $15 \mathrm{~cm}$ in one of the two transverse planes, and also the subsequent high telescopic index of 4 in this plane. This necessitated in particular the deployment of a new type of knob [23] to mimic stand-alone quadrupoles in the arcs (orbit bump in lattice sextupoles, see Fig. 12). On the other hand, the triplet aperture was found to be as expected in this configuration (about $9.5 \sigma$ for a full crossing angle of $260 \mu \mathrm{rad}$ [9]), leaving enough room for adjusting the TCTs. As soon as the new TCT settings were approved by the LHC operation, loss map measurements successfully took place, together with an asynchronous dump test, which did not show any bad surprises and gave the green light for an intensity ramp up.

61 nominal bunches (with one noncolliding, and including a train of 48 bunches) were injected, ramped, and collided in this new machine configuration. After a 


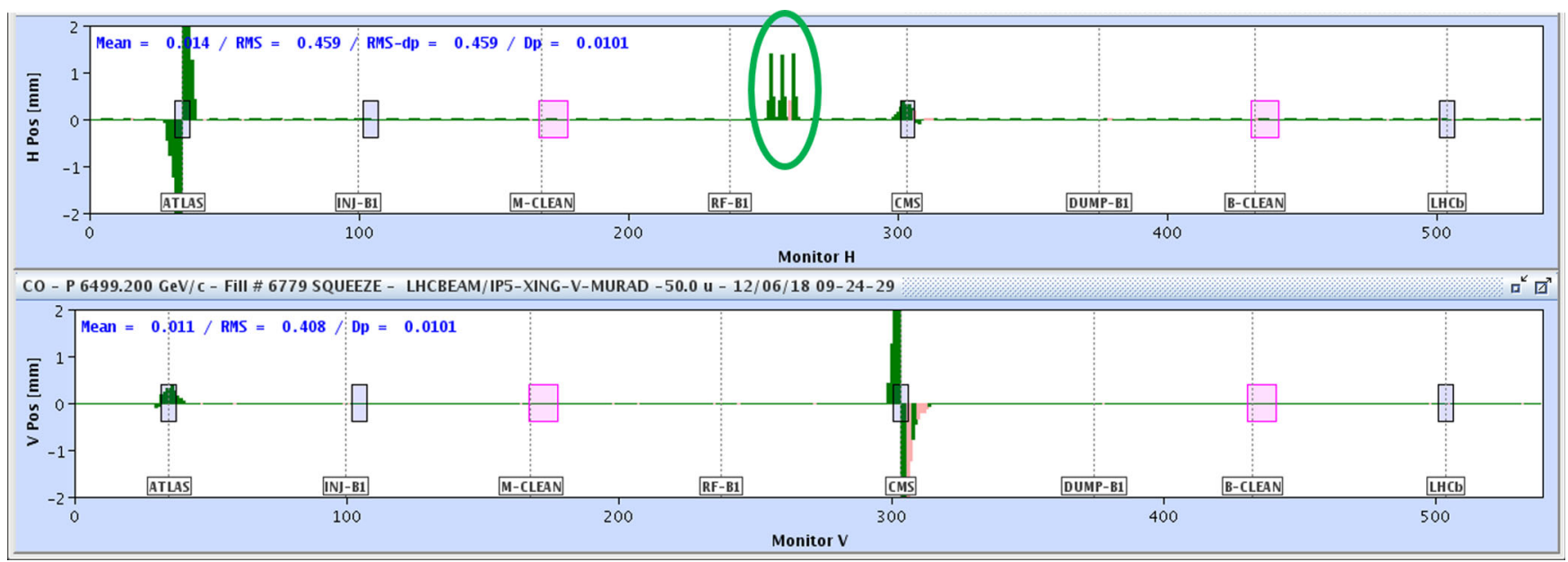

FIG. 11. 2018 flat ATS MD: closed orbit measured for Beam 1 (against a ref. flat orbit) after optics correction. The crossing plane is horizontal in ATLAS and vertical in CMS, i.e., rotated by $90 \mathrm{deg}$. with respect to the nominal configuration. A new type of $\beta$-beating knob ( $3 \pi \mathrm{H}$ orbit bump) is deployed in sector 45.

reoptimization of the working point along the diagonal (to compensate for the BBLR induced tune shift which is not zero in the case of flat optics), switching the MO polarity to negative helped to reach the burn-off limit, for various crossing angles from $260 \mu \mathrm{rad}$ down to $200 \mu \mathrm{rad}$ (8.2-10.6 $\sigma$ for $\gamma \epsilon=2.5 \mu \mathrm{m})$ and a bunch population in the range of $0.9-1 \times 10^{11} \mathrm{p} / \mathrm{b}$ in average (see Fig. 13). The mitigation of the BBLR effect with octupoles is however expected to be suboptimal at higher beam intensity, typically in the range of $1.5 \times 10^{11} \mathrm{p} / \mathrm{b}$ which is relevant for HL-LHC. In order to keep a crossing angle as low as $10 \sigma_{X}$ in this intensity range, dedicated new hardware, namely DC current wires, are needed (see, e.g., [24,25] for a description of the first LHC wire prototypes and first test results obtained at the LHC, and [26] for a comparison between the two BBLR mitigation techniques, namely wire and octupole, in the context of HL-LHC flat optics). The specific luminosity was recorded to be about $25 \%$ higher than for the nominal collision optics (see Fig. 14), as expected due to the sizable mitigation of the geometric loss factor. More results from this MD program can be found in [28].

\section{SUMMARY AND OUTLOOK FOR RUN 3}

The HL-LHC baseline optics fully relies on the ATS scheme, firstly in terms of low- $\beta^{*}$ optics feasibility. The successful early implementation of the scheme in the LHC (2017), but with still mildly telescopic optics, together with dedicated high-intensity beam tests in special machine configurations which took place in Run 2, give a very strong confidence in the viability of the ATS mechanics and

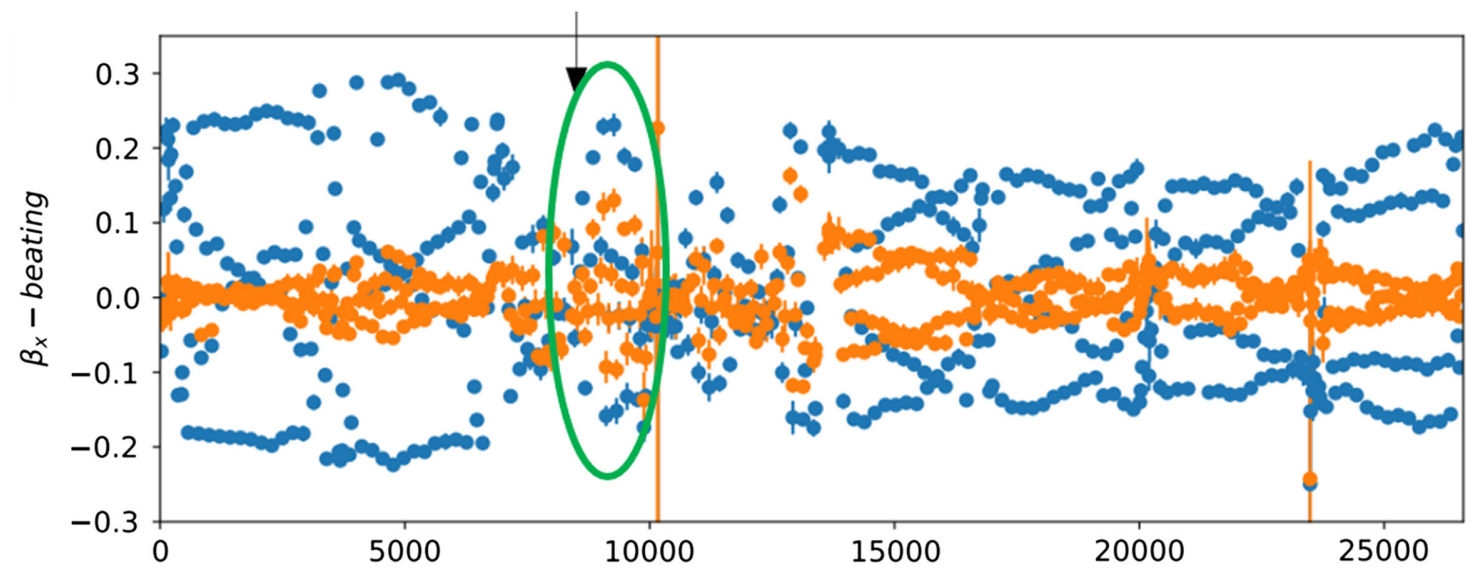

FIG. 12. $\beta$-beating [\%] measured before and after correction at $\beta_{\|, X}^{*}=(60,15) \mathrm{cm}$ (case of Beam1H). The $\beta$-beating at mid-arc in sector 45 (emphasized by the green oval) is improved thanks to an orbit bump and the resulting quadrupolar feed-down effects from the lattice sextupoles (knowing that no standalone quadrupoles is available at this ring location). 


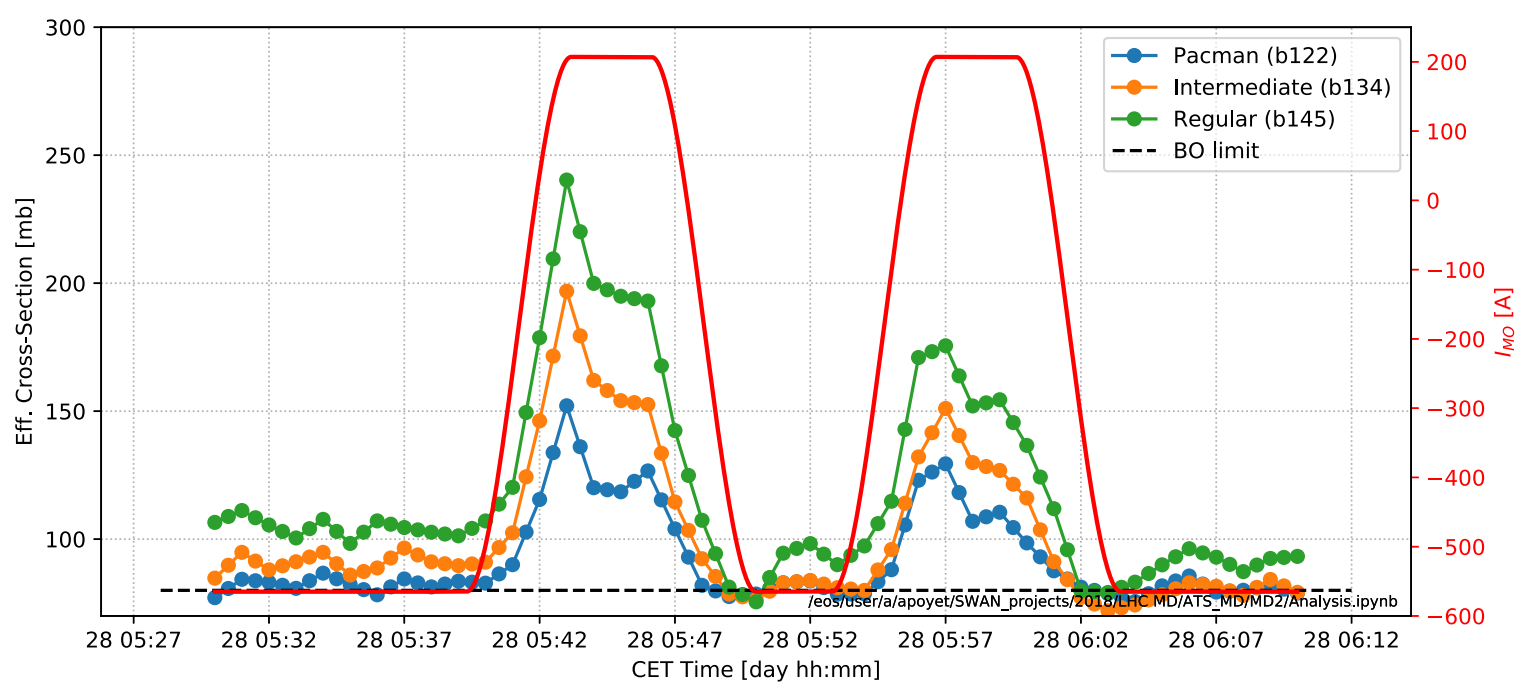

FIG. 13. 2018 flat ATS MD: lifetime (effective cross-section) vs MO current measured for a few selected bunches of Beam 1 with different BBLR collision schedule (full crossing angle of $200 \mu \mathrm{rad}$ ). The machine was filled with $48+12+1=61$ nominal bunches, in particular a train of 48 bunches colliding at IP1 and IP5. During the MO scan, the Beam 2 bunch population was measured in the range of $0.9-1.0 \times 10^{11} \mathrm{p} / \mathrm{b}$.

of its by-products. In particular, an excellent optics correctability was demonstrated with a telescopic index of up to 3 , beyond which some (a priori still manageable) difficulties start to show up. Loss maps were measured using ATS optics with HL-LHC-like telescopic indexes of up to 3-4, for round and flat optics, and were found very similar to those obtained with the nominal optics. As a result, for each ATS optics configuration studied in Run 2 (see summary in Table I), high intensity beam tests were systematically authorized by the LHC Machine Protection Panel, using filling scheme containing ultimately a few or many trains of nominal bunches (depending on the final goal of each ATS MD program).

\begin{tabular}{|c|c|c|c|c|}
\hline 28-Jul-2018 03:45:37 & Fill \#: 6995 & Energy: $6499 \mathrm{GeV}$ & I(B1): $6.32 \mathrm{e}+12$ & $\mid(B 2): 6.36 \mathrm{e}+12$ \\
\hline & ATLAS & ALICE & CMS & LHCb \\
\hline Experiment Status & STANDBY & STANDBY & STANDBY & STANDBY \\
\hline Instantaneous Lumi [(ub.s) $\wedge-1]$ & 480.230 & 0.000 & 495.734 & 0.011 \\
\hline BRAN Luminosity [(ub.s) $\lambda-1]$ & 609.8 & 0.0 & 432.8 & 0.0 \\
\hline Fill Luminosity (nb)^-1 & 0.000 & 0.000 & 0.000 & 14979.054 \\
\hline Beam 1 BKCD & 0.000 & 0.000 & 2.324 & 0.000 \\
\hline Beam 2 BKCD & 0.000 & 0.000 & 0.890 & 0.021 \\
\hline Beta* & $0.65 \mathrm{~m}$ & $10.00 \mathrm{~m}$ & $0.65 \mathrm{~m}$ & $3.00 \mathrm{~m}$ \\
\hline Crossing Angle (urad) & $o(v)$ & $200(n)$ & $O(H)$ & $-250(\mathrm{H})$ \\
\hline LHCb VELO Position DU & $\mathrm{p}:-0.0 \mathrm{~mm}$ & ADJUST & TOTEN & CALBRATION \\
\hline
\end{tabular}

FIG. 14. 2018 flat ATS MD: First collision of bunch trains with flat optics $\left(12+48\right.$ colliding bunches with $\left\langle N_{p}\right\rangle=1.03 \times$ $\left.10^{11} \mathrm{p} / \mathrm{b}\right)$. The luminosity was approaching $5 \times 10^{32} \mathrm{~cm}^{-2} \mathrm{~s}^{-1}$ in ATLAS and CMS (corresponding to $10^{35} \mathrm{~cm}^{-2} \mathrm{~s}^{-1}$ for 2748 colliding bunches and $2.2 \times 10^{11} \mathrm{p} / \mathrm{b}$ ). This picture, taken during the MD to keep record of the first collisions of bunch trains with flat optics at the LHC, is a snapshot of the so-called "LHC page 1" [27], where the main beam, optics and luminosity related parameters are dynamically published during nominal operation.
In addition, an extension of the existing combined ramp and squeeze concept, where the telescope is deployed already in the ramp, demonstrated with high brightness beams (and up to 800 bunches) that the ATS scheme offers as well a very powerful knob for mitigating possible intensity limitations coming from the machine impedance. First high-intensity beam tests took place using a very special LHC hypercycle, leading to a flat collision optics compatible with the aperture of the existing triplet, although with a $\beta^{*}$ as small as the HL-LHC baseline $\beta^{*}$ of $15 \mathrm{~cm}$ in one of the two transverse planes, and set to $60 \mathrm{~cm}$ in the crossing plane. A minimal full crossing angle of $200 \mu \mathrm{rad}$ (unexpectedly low) was also demonstrated in this configuration, for a bunch population still in the range of $10^{11} \mathrm{p} / \mathrm{b}$, but only relying on the existing lattice octupoles in order to mitigate the long-range beam-beam interactions which, otherwise, become severely detrimental to the beam lifetime for such optics parameters. With such optics parameters, the geometric luminosity loss factor is around 0.89 (taking $\gamma \epsilon=2.5 \mu \mathrm{m}$ and $\sigma_{z}=7.5 \mathrm{~cm}$ for the r.m.s. bunch length), which could be directly compared to 0.68 obtained with the "equivalent" round ATS optics used for operating the LHC in 2017 and 2018 (i.e., with $\beta^{*}=\sqrt{15 \times 60}=30 \mathrm{~cm}$, but a full crossing angle in the range of $300 \mu \mathrm{rad}[16])$.

HL-LHC-like telescopic optics can de facto be considered as immediately available in order to extract the best possible LHC performance from the beam intensity ramp up which is planned to gradually take place in Run 3 in the framework of the LHC Injector Upgrade (LIU) project [29]. Another strong motivation is to warrant as well the smoothest possible transition between the two machines in terms of beam parameters, optics, but also 
TABLE I. Summary of the various ATS optics configurations demonstrated so far in operation and in dedicated machine experiments: most relevant optics parameters at the end of ramp (EoR) and end of squeeze (EoS), typical beam intensity used, and main by-product.

\begin{tabular}{|c|c|c|c|c|c|c|}
\hline Year & Optics type & $r^{\text {Tele }}$ EoR & $r^{\text {Tele }}$ EoS & $\beta_{\text {Tele }}^{*}[\mathrm{~cm}]$ EoS & $\begin{array}{c}\text { Number of protons } \\
\text { per beam }\end{array}$ & By-product \\
\hline \multicolumn{7}{|c|}{ Nominal operation } \\
\hline 2017 & Round [14] & 1.0 & 1.33 & 30 & $2.5-3.0 \times 10^{14}$ & $\beta^{*}$ reduction beyond IR $1 / 5$ \\
\hline 2018 & Round [14] & 1.0 & 1.60 & 25 & $2.5-3.0 \times 10^{14}$ & Matching quadrupole limit \\
\hline \multicolumn{7}{|c|}{ Dedicated beam experiments } \\
\hline 2012 & Round [12] & 1.0 & 4.0 & 10 & $\lesssim 10^{10}$ & ATS mechanics demonstration \\
\hline 2016 & Round [14] & 1.0 & $1.2 \rightarrow 1.9$ & $33 \rightarrow 21$ & $\lesssim 3 \times 10^{11}$ & Machine validation (collimation) \\
\hline 2017 & Round [18] & 1.0 & 2.9 & 35 & $1.7 \times 10^{13}$ & BBLR mitigation with octupoles \\
\hline 2018 & Round [20] & 3.1 & 3.1 & 65 & $1.3 \times 10^{14}$ & MO instability threshold reduction \\
\hline 2018 & Flat [28] & $1.0 / 1.0$ & $1.0 / 4.0$ & $60 / 15$ & $6.5 \times 10^{12}$ & Luminosity loss factor mitigation \\
\hline
\end{tabular}

dedicated beam manipulations such as $\beta^{*}$-levelling over a very large dynamic range (aiming at preserving the luminosity during physics data taking, despite of the proton burn-off, by dynamically squeezing $\beta^{*}$ in collision). From this perspective, a dedicated working group was set up in 2018 in order to conceive, prove the feasibility, and validate on paper appropriate machine configurations for operating the machine in Run 3. The first conclusions of the working group are summarized below [30].

$\beta^{*}$-levelling range. With a levelled luminosity limited to $\sim 2 \times 10^{34} \mathrm{~cm}^{-2} \mathrm{~s}^{-1}$ by the cryo-cooling capacity of the existing inner triplets [31], and a bunch population at SPS extraction of up to $1.8 \times 10^{11}$ at the end of 2022 (at the limit, but a priori still compatible with most of the LHC sub-systems), the $\beta^{*}$ levelling range might reach a factor 4 to 5 toward the end of Run 3 (compared to only 20\% demonstrated in operation in 2018), from $\beta^{*} \gtrsim 1 \mathrm{~m}$ down to $\sim 25 \mathrm{~cm}$.

$\beta^{*}$-leveling strategy. Following a strong preference from the forward physics (FP) experiments (AFP [32] and PPS [33]) to work with a $\beta^{*}$-independent transport $R$-matrix from the IP to the roman pots (in between Q5 and Q6), the telescopic squeezing mode (by acting externally on the matching quadrupoles in IR8/2/4/6) is clearly the best strategy for $\beta^{*}$-levelling.

Antitelescopic optics. In telescopic squeezing mode, the $\beta^{*}$ and tele-index relative variations are strictly the same by construction. From ATS MD experience in Run 2, the telescopic index is however recommended not to exceed 3 at the end of the luminosity levelling period, namely $r_{\text {end }}^{\text {Tele }} \lesssim 3$. This target is however still too restrictive compared to a $\beta^{*}$ leveling range of 5. Starting the $\beta^{*}$-leveling beam process with an antitelescopic optics deployed earlier in the ramp, that is with $r_{\text {start }}^{\text {Tele }}<1$, seems de facto be the only way to go in order to fulfill all the constraints $\left(r_{\text {end }}^{\text {Tele }} / r_{\text {start }}^{\text {Tele }} \sim 5\right.$ and $r_{\text {end }}^{\text {Tele }} \lesssim 3$ ).
Telescopic index management. Adjusting the end of ramp telescopic index to $r_{\text {start }}^{\text {Tele }} \sim 1 / 2$ (i.e., $r_{\text {end }}^{\text {Tele }} \sim 2.5$ for a $\beta^{*}$-leveling range of 5 deployed exclusively in telescopic mode) is also found to strongly minimize the risk of running out of strength the lattice octupoles, up to a beam energy of $7 \mathrm{TeV}$ and a brightness of 1 (i.e., $1.8 \times 10^{11} \mathrm{p} / \mathrm{b}$ within a normalized emittance of $\gamma \epsilon=1.8 \mu \mathrm{m}$, corresponding to the best possible beam quality at flat-top energy expected in Run 3).

Presqueezed $\beta^{*}$ management. The last parameter to be determined is the value of the presqueezed $\beta^{*}$ at the end of the ramp, keeping in mind that the corresponding IR1 and IR5 matching quadrupole settings are then kept constant during $\beta^{*}$-levelling in telescopic squeezing mode. For a typical $\beta^{*}$ reach of $\beta_{\min }^{*}=25 \mathrm{~cm}$ demonstrated in Run 2 (limited by the aperture of the existing triplet at minimal crossing angle), and based on the above discussion, one gets $\beta_{\mathrm{Pre}}^{*}=r_{\text {end }}^{\text {Tele }} \times \beta_{\min }^{*} \sim$ $60 \mathrm{~cm}$, which is substantially larger than the minimum possible presqueezed $\beta^{*}$ of $36 \mathrm{~cm}$ in the LHC (see Sec. I A). Under these conditions, certain optics constraints, internal to the insertions 1 and 5 , can be properly treated, such as a maximization of the normalised dispersion at the roman pots aiming at reducing the minimum mass detection threshold which is accessible by the FP experiments.

To summarize, the possibilities offered by the ATS scheme go well beyond the effective realization of advanced collision optics with decimetric $\beta^{*}$ values for the (HL-)LHC. Other ATS by-products are planned to be carefully exploited. Deployed at full scale in Run 3, the ATS scheme should in particular not only facilitate the calibration, but also maximize the discovery potential of the forward physics experiments. Going in this direction, the machine configuration is also planned to be correctly dimensioned (in terms of telescopic index), in order to take advantage as much as possible of the LIU beam intensity ramp up, for further improving the LHC performance, while soundly preparing the ground for the HL-LHC. 


\section{ACKNOWLEDGMENTS}

The successful beam tests with ATS optics obviously required the joint and coordinated effort of many different LHC teams, in particular the LHC Operation team, but also the Optics Measurement and Correction, the Collimation, the Beam-Beam, the Instability, the Transverse Damper (ADT) and the LHC Beam Dump (LBDS) teams, without forgetting the constant support and advice from the LHC Machine Protection Panel, the LHC MD Coordination, and from the Management of the CERN Accelerator and Technology Sector, in particular: M. Albert, G. Arduini, Y. Le Borgne, C. Bracco, R. Bruce, X. Buffat, F. Carlier, P. Collier, A. Garcia-Tabares, K. Fuchsberger, R. Giachino, N. Karastathis, M. Lamont, E. Maclean, L. Malina, E. Métral, D. Mirarchi, N. Mounet, D. Nisbet, L. Normann, G. Papotti, T. Persson, M. Pojer, L. Ponce, Y. Papaphilippou, S. Redaelli, B. Salvachua, P. Skowronski, G. Sterbini, R. Suykerbuyk, R. Tomas, J. Uythoven, D. Valuch, A. Wegscheider, D. Wollmann, and, very likely, many others. Finally I would like to express my gratitude to the two referees for the accurate and patient work of proofreading and commenting on the draft manuscript of this article.

[1] S. Fartoukh, Optics challenges and solutions for the LHC Insertion Upgrade Phase I, in Chamonix 2010 Workshop on LHC Performance, Chamonix, France, 25-29 Jan 2010, Report No. CERN-ATS-2010-026, pp. 262-290, 2010.

[2] S. Fartoukh, Towards the LHC Upgrade using the LHC well-characterized technology, CERN Report No. SLHCProject-Report 49, 2010.

[3] S. Fartoukh, Breaching the Phase I optics limitations for the HL-LHC, in Chamonix 2011 Workshop on LHC Performance, Chamonix, France, 24-28 Jan 2011, Report No. CERN-ATS-2011-005, pp. 302-316, 2011.

[4] S. Fartoukh, Achromatic telescopic squeezing Scheme and its application to the LHC and its luminosity upgrade, Phys. Rev. Accel. Beams 16, 111002 (2013).

[5] O. Brüning, P. Collier, P. Lebrun, S. Myers, R. Ostojic, J. Pool, and P. Proudlock, LHC Design Report, v.1: the LHC Main Ring, CERN Report No. CERN-2004-003-V-1, 2004, https://cds.cern.ch/record/782076.

[6] G. Apollinari, I. Bejar, O. Bruning, P. Fessia, M. Lamont, L. Rossi, and L. Tavian (Ed.), High-Luminosity Large Hadron Collider (HL-LHC): Technical Design Report V. 0.1, CERN Report No. CERN-2017-007-M, 2017, http:// cds.cern.ch/record/2284929.

[7] D. Brandt and M. Lamont, Commissioning of the high energy combined ramp and squeeze, CERN Report No. SL-Note-98-070-MD, 1998.

[8] M. Solfaroli, S. Redaelli, R. Tomas, and J. Wenninger, Combined ramp and squeeze to $6.5 \mathrm{TeV}$ in the LHC, in Proc. 7th International Particle Accelerator Conference 2016, Busan, Korea, 8-13 May 2016 (JACoW, Geneva, 2016), pp. 1509.
[9] S. Fartoukh, N. Karastathis, M. Solfaroli, and R. Tomas, About flat telescopic optics for the future operation of the LHC, CERN Report No. CERN-ACC-2018-0018, 2018.

[10] X. Buffat et al., Transverse instabilities, Proc. 10th Evian Workshop on LHC beam operation, Evian Les Bains, France, 30 Jan-1 Feb. 2019, Report No. CERN-ACC2019-059, pp. 233-243, 2019.

[11] S. Fartoukh, First demonstration with beam of the achromatic telescopic squeezing (ATS) scheme, in Chamonix 2012 Workshop on LHC Performance, Chamonix, France, 6-10 Feb 2012, Report No. CERN-ATS-2012-069, pp. 128-134, 2012

[12] S. Fartoukh et al., The $10 \mathrm{~cm}$ beta* ATS MD, CERN Report No. CERN-ATS-Note-2013-004 MD, 2013.

[13] R. Bruce, C. Bracco, R. De Maria, M. Giovannozzi, A. Mereghetti, D. Mirarchi, S. Redaelli, E. Quaranta, and B. Salvachua, Reaching record-low $\beta^{*}$ at the CERN Large Hadron Collider using a novel scheme of collimator settings and optics, in Nucl. Instrum. Methods Phys. Res., Sect. A 848, 19 (2017).

[14] S. Fartoukh, Experience with ATS optics, in Proc. 7th Evian Workshop on LHC beam operation, Evian Les Bains, France, 13-15 December 2016, Report No. CERN-ACC-2017-094, pp. 93-103, 2017.

[15] D. Pellegrini, S. Fartoukh, N. Karastathis, and Y. Papaphilippou, Multiparametric response of the LHC dynamic aperture in presence of beam-beam effects, J. Phys. Conf. Ser. 874, 012007 (2017).

[16] J. Wenninger, Operation and configuration of the LHC in Run 2, CERN Report No. CERN-ACC-NOTE-2019-0007, 2019.

[17] S. Fartoukh, ATS optics for HL: what is still missed?, Presented in Chamonix 2018 Workshop on LHC Performance, Chamonix, France, 29 Jan-2 Feb 2018 (unpublished), https://indico.cern.ch/event/676124/ contributions/2768616/.

[18] S. Fartoukh et al., Round optics with large telescopic index, CERN Report No. CERN-ACC-2018-0032, 2018.

[19] H. Damerau et al., LIU: Exploring alternative ideas, Review of LHC and Injector Upgrade Plans Workshop, Archamps, France, 29-31 October 2013 (unpublished), https://indico.cern.ch/event/260492/.

[20] S. Fartoukh et al., Combined ramp and telescopic squeeze, CERN Report No. CERN-ACC-2020-0028, 2020.

[21] S. Fartoukh, A. Valishev, and D. Shatilov, An alternative high luminosity LHC with flat optics and long-range beambeam compensation, in Proc. 5th International Particle Accelerator Conference 2015, Richmond, VA, USA, May 3-8 2015 (JACoW, Geneva, 2015).

[22] S. Fartoukh, A. Valishev, Y. Papaphilippou, and D. Shatilov, Compensation of the long-range beam-beam interactions as a path towards new configurations for the high luminosity LHC, Phys. Rev. Accel. Beams 18, 121001 (2015).

[23] J. C. de Portugal, R. Tomas, and M. Hofer, New local optics measurements and correction techniques for the LHC and its luminosity upgrade, Phys. Rev. Accel. Beams 23, 041001 (2020).

[24] A. Rossi et al., Progress with long-range beam-beam compensation studies for high luminosity LHC, in Proc. 8th International Particle Accelerator Conference 2017, 
Copenhagen, Denmark, May 14-19, 2017 (JACoW, Geneva, 2017).

[25] G. Sterbini et al., First results of the compensation of the beam-beam effect with dc wires in the LHC, in Proc. 10th International Particle Accelerator Conference 2019, Melbourne, Australia, May 19-24, 2019 (JACoW, Geneva, 2019).

[26] S. Fartoukh, N. Karastathis, and M. Solfaroli, Flat optics for (HL-)LHC, Presented in 8th HL-LHC Collaboration Meeting, CERN, 15-18 October 2018 (unpublished), https://indico.cern.ch/event/742082/.

[27] https://op-webtools.web.cern.ch/vistar/vistars.php? usr=LHC1.

[28] S. Fartoukh et al., First high-intensity beam tests with telescopic flat optics at the LHC, CERN Report No. CERN-ACC-2019-0052, 2019.
[29] H. Damerau et al., LHC injectors upgrade: Technical design report No. CERN-ACC-2014-0337, 2014.

[30] N. Karastathis et al., LHC Run-III configuration working group report, in Proc., 10th Evian Workshop on LHC beam operation, Evian Les Bains, France, 30 Jan-1 Feb. 2019, Report No. CERN-ACC-2019-059, pp. 273-284, 2019.

[31] G. Ferlin et al., Cryogenics experience during Run2 and impact of LS2 on next run, in Proc., 10th Evian Workshop on LHC beam operation, Evian Les Bains, France, 30 Jan-1 Feb. 2019, Report No. CERN-ACC2019-059, pp. 85-90, 2019.

[32] R. Staszewski et al. (AFP Collaboration), The AFP Project, Acta Phys. Pol. B 42, 1615 (2011).

[33] M. Albrow et al. (CMS and TOTEM Collaborations), CMS-TOTEM precision proton spectrometer, CERN Reports No. TOTEM-TDR-003, No. CMS-TDR-13, 2014. 